\title{
CAEP/ACMU 2014 Scientific Abstracts Keyword Index
}

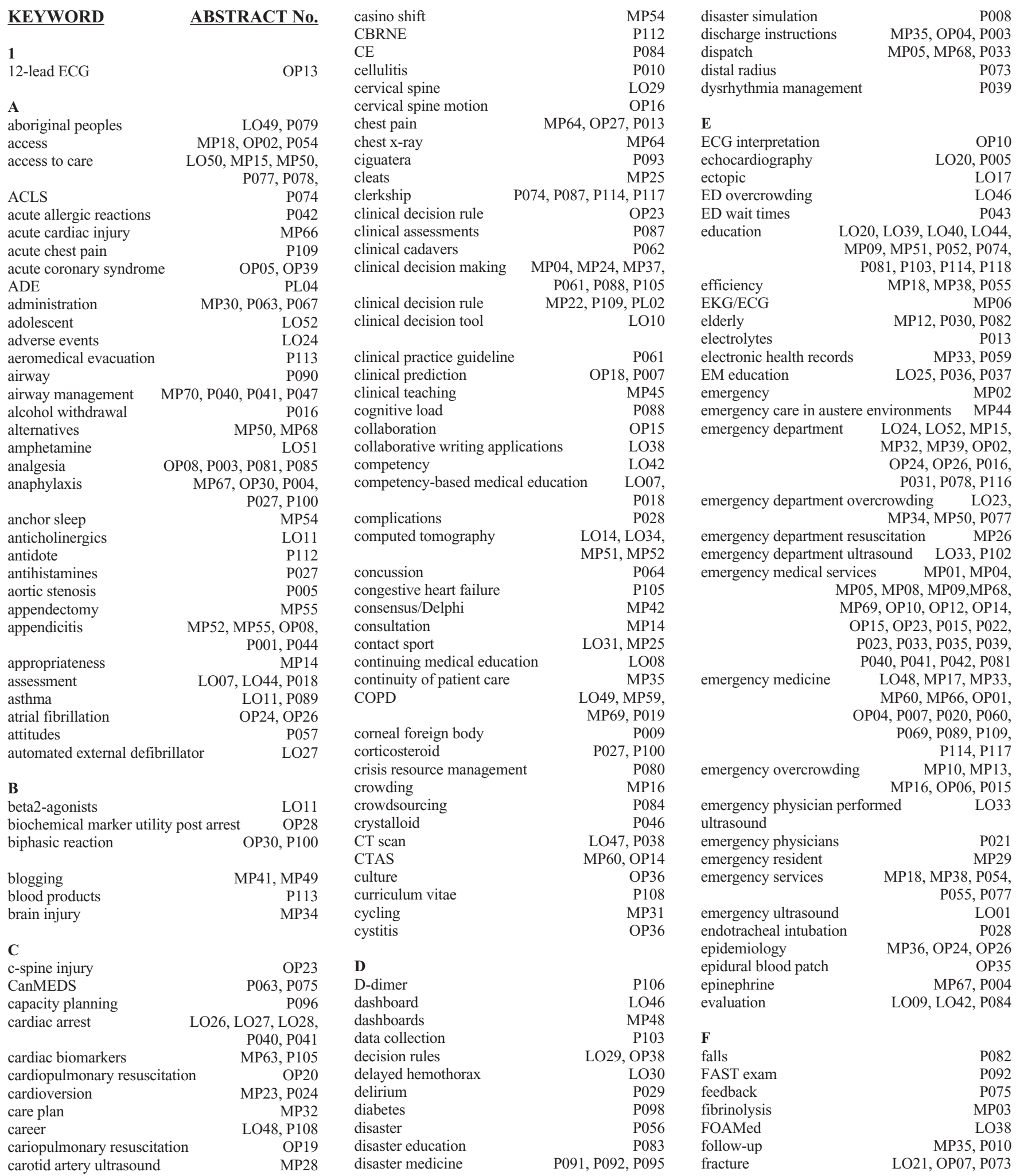




\begin{tabular}{|c|c|c|c|c|c|}
\hline frailty & LO13 & investigations & MP64 & $\mathbf{P}$ & \\
\hline frequent emergency department & LO49, P072 & IV cannulation & MP09 & pain & LO37, MP02, OP34 \\
\hline users & & IV therapy & P010 & pain management & LO36, OP33, P003, \\
\hline frostbite & P032 & & & & 25, P058, P099, P115 \\
\hline functional decline & MP21, MP39 & $\mathbf{J}$ & & paramedics & MP02, MP04, MP06, \\
\hline functional limitation & OP29 & journal club & MP49 & & MP67, OP22 \\
\hline funding & P104 & & & patient flow & LO19, LO47, P110 \\
\hline & & $\mathbf{K}$ & & patient perception & MP20 \\
\hline G & & ketamine & MP19 & patient safety & LO43, MP30, OP22, \\
\hline gender differences in post arrest care & OP21 & ketamine-propofol & LO35 & & P026, P051 \\
\hline geriatric emergency medicine & LO08 & knowledge needs & P097 & patient satisfaction & P058 \\
\hline geriatrics & LO13, MP15, & knowledge translation & 50, P078 & pediatric emergency & OP34, P025 \\
\hline & MP39 & & & ediatric emergency medicin & OP33, P098 \\
\hline global health & P052 & $\mathbf{L}$ & & pediatrics & MP27, MP52, MP71, \\
\hline guidelines & 9, P049, P098 & laboratory testing & $\begin{array}{r}\text { MP17 } \\
\text { P080 }\end{array}$ & OP07, & 7, ОР08, ОР09, ОР30, \\
\hline & & leadership & P080 & OP3 & 31, P001, P004, P038, \\
\hline $\mathbf{H}$ & & length of stay & 044, P079 & & 011,P090, P097, P115 \\
\hline handover & P026 & long-term care & 15, P110 & eer review & LO03 \\
\hline hazardous drinking & P116 & cture & P071 & ady & OP33 \\
\hline HazMat & P112 & LWBS & P079 & erformance & MP48 \\
\hline head injury & P065 & & & ericardiocentesis & P094 \\
\hline LO14, LO34, & 4, P038, P071 & $\mathbf{M}$ & & eripheral vertigo & LO04 \\
\hline ealth education & MP20 & lajor trauma & 57, PL03 & phone follow-up & LO24 \\
\hline health indicators & LO06 & lar fractures & MP56 & hysi & LO45 \\
\hline health information technology & OP12 & ualty & P091 & physician-patient interaction & OP04 \\
\hline health literacy & LO05 & ass casualty incident & MP08 & point-of-care ultrasound & PL01, LO09, \\
\hline health policy & LO23, MP37 & thering & P083 & & LO22, LO33, MP65, \\
\hline health promotion & P034 & ering medicine & LO02 & & OP03, P050, P102 \\
\hline heart failure & OP38 & norrhages & 39, MP11 & police & $\mathrm{OP} 03$ \\
\hline heavy emergency department users & MP32 & medical education & 41, LO42, & olicy & P037 \\
\hline helmet & MP31 & LO43, MP46, P0 & 69, Р070, & population studies & OP39 \\
\hline hemorrhage control & MP01 & P075, P0 & 087, P088 & post-arrest care & OP28 \\
\hline high $\mathrm{c}$ & LO53 & edical informatics & , P031 & post-cardiac arrest care & LO26 \\
\hline sitivity cardiac troponin & MP66 & LO40, MP & 47, P012 & ost-concussion symptoms & MP53, OP18 \\
\hline homel & MP34 & LO & 19, P060 & post-dural puncture headache & OP35, P071 \\
\hline admission & $\mathrm{P} 030$ & lth apprehension & OP03 & post-intubation hemodynami & ic instability \\
\hline lunteers & P104 & atus & MP12 & postgraduate education & LO36, P067 \\
\hline hyperte & P021 & 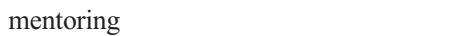 & MP45 & posttraumatic stress disorder & P076 \\
\hline hypotension & PL01 & injury & MP25 & practice patterns & LO16, P021 \\
\hline & & mild traumatic brain injury & 2, MP53, & practice variation & LO18, MP40 \\
\hline & & & OP18 & pre-hospital & $3, \mathrm{OP} 13$ \\
\hline LO15, LO18, M & MP24, MP40, & minor head injury & MP27 & pre-hospital care & MP07, OP11, \\
\hline MP51, MP56, N & MP65, P001, & LO1 & 13, MP21 & & P035, P099 \\
\hline & P044, P106 & cic injury & 30, OP29 & al management & OP16 \\
\hline imaging - computed tomography & LO19, P006 & sis & OP39 & & LO17 \\
\hline immobilzation & P002 & 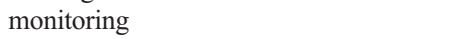 & MP59 & Iness & P056 \\
\hline ntation science network & OP21 & ting & P036 & prevention & P034 \\
\hline implications for teaching & P028 & mortality & P072 & procedural sedation & LO35, MP70, \\
\hline in situ simulation & LO39 & & & & OP32, P045 \\
\hline infants & LO37 & $\mathbf{N}$ & & prod & P118 \\
\hline$n$ & P020 & itis & P020 & & LO35 \\
\hline information sharing & MP26 & sessment & MP33 & prothrombin time & OP27 \\
\hline MP31, & , OP17, P082 & ADES & PL04 & & P060 \\
\hline y prevention & OP01 & ock & MP & hosis & LO51 \\
\hline inner city & P057 & k City & MP29 & blic health & P093 \\
\hline innovations in EM education & LO01, LO02, & cerin & MP07 & put & P043 \\
\hline LO03, LO07, L & LO08, LO09, & nursing & LO29 & pulmonary embolism & MP62, P106 \\
\hline MP41, MP42, M & MP43, MP44, & & & & \\
\hline MP45, MP46, M & MP47, MP48, & O & & & \\
\hline MP49, OP11, OP25, I & , P008, P009, & sonography & LO32 & & LO03, MP30 \\
\hline P012, P057, P059, & 9,P062, P063, & ucational resources & LO41 & quality improvement & LO47, MP17, OP06, \\
\hline P068, P083, P086, & 6, P108, P117 & & P086 & OP3 & 37, P019, P025, P058, \\
\hline INR & OP27 & liameter & LO32 & & P059, P107, P110 \\
\hline interdisciplinary team training & MP11, P080 & t solutions & LO37 & quality indicator & LO23, OP37 \\
\hline internation medicine & MP43 & Ottawa Ankle Rules & LO12 & & \\
\hline P035, & 5, P067, P102 & out-of-hospital emergency medical care & LO02 & $\mathbf{K}$ & \\
\hline international medicine & P068 & 0 & P093 & radius fracture & P002 \\
\hline interprofessional and interdisciplinary & P008 & assessment & P072 & RAFF & P024 \\
\hline intoxication & $\mathrm{LO}_{2}$ & & P065 & andomized controlled trial & OP01, OP09 \\
\hline intracranial pressure & LO32, MP19 & LO12, MP & 16, P043 & id assessment zone & MP10 \\
\hline intralipid & LO53 & overdose & LO53 & rapid sequence intubation & MP19 \\
\hline
\end{tabular}




remifentanil
renal colic
research
residence type
residency education
resident duty hours
resident trainee
resident
resource utilization
respiratory distress
resuscitation
resuscitation guidelines
return of spontaneous circ
reveal
risk Scale
routine investigation
rural
S
safety
satisfaction
screening tools
search engine
sedation
self-reporting
seniors
sepsis
shiftwork
shock
shoulder dislocation
signs and symptoms
simulation

OP32
P006, P007, P050, P085
P103
P030
OP11, P018
LO25, P036, P037
MP37
P070
LO51
P022, P023
OP21, OP28
LO28
P064
OP38
P013
OP20, OP31, P107

LO21, LO25, MP29

P115
MP12, P076
P086

MP23, P024
OP22

MP21, P029

OP37, P107

MP54

OP25, PL01

LO10

$\mathrm{P} 042$

LO01, LO44, MP11,

MP42, MP43, P062, P070,

P091, P094, P095, P096 slit lamp

smoking cessation

social media

socio-economic status

specialist consultation

spinal cord injury

STEMI

stroke

stroke prevention clinic

subarachnoid hemorrhage

superficial thrombophlebitis

suprventricular tachycardia

surgical emergencies

survival

suspected neck injury

symptoms

systematic Review

PP20, P034
LO38, MP41, MP46
P116
OP35
MP36

O03, MP06, OP10, OP13
O04, LO05, LO06, P049
MP28
PL02, LO14, LO34
MP61
P039
MP14
OP19, OP20
OP16
P064
Oia

T

tachycardia

tactical emergency medicine

targeted temperature management

teaching

team error

teamwork

technology

telemetry

therapeutic hypothermia

throughput

tiered response

tongue

transesophageal

transfer

transfusion
MP07

MP44

LO26

P056, P118

$\mathrm{P} 051$

MP26

OP09

MP59

LO28

MP10, MP13

P033

P090

LO20

MP57, PL03

P101, P113 transient ischemic attack

MP28

transport

MP58

trauma

MP01, MP27, MP58, P045, P051, P065, P068, P069,

P073, P099, P101

trauma care organization MP57, PL03

treatment MP61, MP62, P032, P066

tremor

triage

$\mathrm{P} 016$

triage nurse

triage physician

trigger tool

TRISS

LO45, MP60, OP06,

OP14, OP34

LO12

MP13

PL04

$\mathbf{U}$

ultrasound

LO15, LO16, LO17, LO21, MP47, MP55, MP65, OP07, OP25, P012, P052, P092, P094, P095

undergraduate medical education $\quad$ LO22 urinary tract infection $\quad$ OP36, P011 utilization LO18, MP24, MP38, MP40, P054

V

venous thromboembolism MP61, P066

volume surge LO46

volunteer

P104

W

waiting times

weight estimation

welfare

work patterns
LO50

OP31

OP03

LO48

\section{CAEP/ACMU 2014 Scientific Abstracts Author Index}

\begin{tabular}{lrl} 
AUTHOR & ABSTRACT No. & Argintaru N \\
\cline { 2 - 3 } A & & $\begin{array}{l}\text { Armstrong A } \\
\text { Armstrong S }\end{array}$ \\
Aarabi P & P016 & Arntfield R \\
Aaron S & OP38 & \\
Abramson BL & OP21 & Arthur AO \\
Abu-Laban RB & MP31 & Assoignon L \\
Adamson M & LO45 & Athaide V \\
Adekoya D & P032 & Atkinson PR \\
Agaybi S & MP09 & \\
Ahmed A & P001 & Atzema CL \\
Ahmed K & P002 & Aufderheide TP \\
Ahmed S & P102 & Austin E \\
Aklilu F & MP25 & Austin M \\
A1 Juma S & P064 & Austin PC \\
Al Kashmiri A & P068 & Azad A \\
Al-Attar M & P097 & Azaj A \\
Albrecht L & LO36 & Azazh A \\
Ali H & P030 \\
Ali S & OP34, P115 & B \\
Allen RA & P003 & Bailey B \\
Alqrashi W & OP30, P004 & Baker L \\
Alzahrani H & P005 & Bandiera G \\
Andruchow J & MP14 & Bank I \\
Archambault PM & P007 & Bardua DJ \\
Archer B & &
\end{tabular}

$\begin{aligned} \text { P006, P085 } & \text { Barratt L } \\ \text { LO29 } & \text { Bastian J } \\ \text { LO29 } & \text { Beaudry P } \\ \text { LO20, LO22, MP47, } & \text { Bednarczyk JM } \\ \text { P012, P050 } & \text { Bégin F } \\ \text { LO02, MP01, MP44, } & \text { Bell R } \\ \text { P039, P040, P041, P042 } & \text { Benjamin S } \\ \text { P072 } & \text { Berman S } \\ \text { MP19 } & \text { Bernklau R } \\ \text { LO48, MP17, P007, } & \text { Betz M } \\ \text { P019, P069, P073, PL01 } & \text { Bhanich Supapol W } \\ \text { LO04, P021 } & \text { Bhatti JA } \\ \text { OP28 } & \text { Bhimani M } \\ \text { MP43 } & \text { Bhutani M } \\ \text { OP22 } & \text { Bidlake E } \\ \text { LO04, P021 } & \text { Bienkowski A } \\ \text { MP25 } & \text { Bierman AS } \\ \text { P067 } & \text { Bilodeau A } \\ \text { MP43, P065, P102 } & \text { Birnie D } \\ & \text { Bishnoi A } \\ & \text { Blackie B } \\ \text { LO29 } & \text { Blake DF } \\ \text { MP48 } & \text { Blake GD } \\ \text { LO47, P058 } & \text { Bloom GA } \\ \text { OP07, P008, P080 } & \text { Blouin D } \\ \text { LO52 } & \text { Bohrer M } \\ \text { OP15 } & \text { Boissy P }\end{aligned}$

OP37, P058, P107

P059

MP59

LO41

MP03

$\mathrm{LO} 23$

P069, P073

P065, P067

$\mathrm{P} 112$

OP06

MP13

MP25

P006, P085

P089

P026

MP04

OP21

LO38

MP23, P024

P118

P075

P009

P009

P064

LO38

OP12

OP16 